\section{Placement of tunnelled silicone haemodialysis
catheters in a haemodialysis
catheters in a radiology
intervention unit radiology
intervention unit}

\section{J Hartlett} MB ChB

E Loggenberg MMedRad (D)

C $\mathrm{S}$ de Vries MMedRad (D)

Department of Diagnostic Radiology University of the Free State

Bloemfontein
As an alternative to surgical placement, tunnelled haemodialysis catheters can be placed in the sterile environment of an interventional unit. The same dress code and access to the unit is used as in a sterile operating theatre.

This was a retrospective study conducted over a 3-year period. Placement was done under real-time ultrasound guidance under sterile conditions. Suitable venous puncture was done and the guide wire manipulated under fluoroscopy. If necessary, a venogram may be used to determine the patency of the vein (Figs 1 - 4) as well as any anatomical variants or displacement by tumours or masses (Fig. 5). The catheter is then tunnelled from a distant position on the anterior abdominal wall and placed in a central vein via a peel-away sheath and the catheters are always manipulated under fluoroscopy to determine the optimal position (Figs 6 - 8).

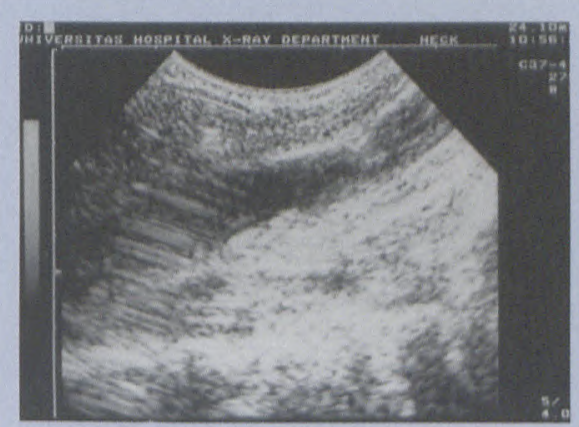

Fig. 1. Internal jugular vein.

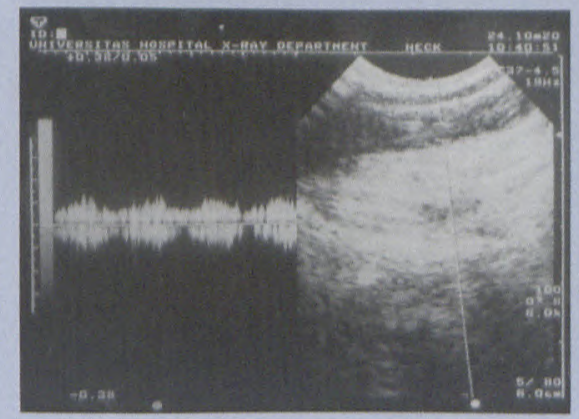

Fig. 2. Doppler flow in internal jugular vein.

After placement in the superior vena cava, free back-flow of blood

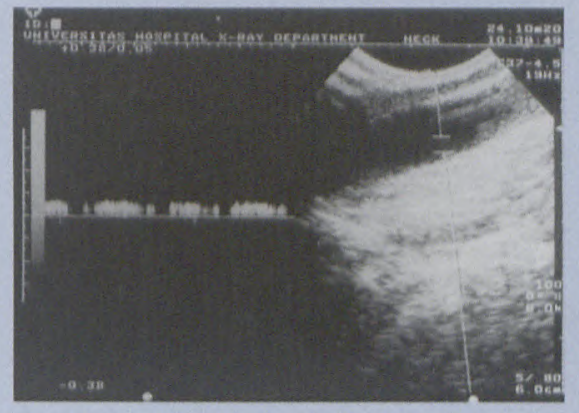

Fig. 3. Determine patency of vein.

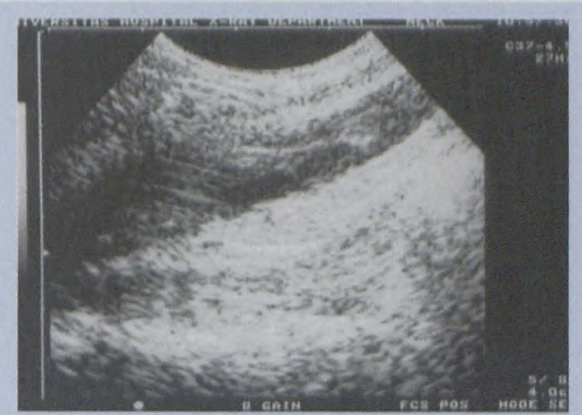

Fig. 4. Needle in internal jugular vein under ultrasound guidance.

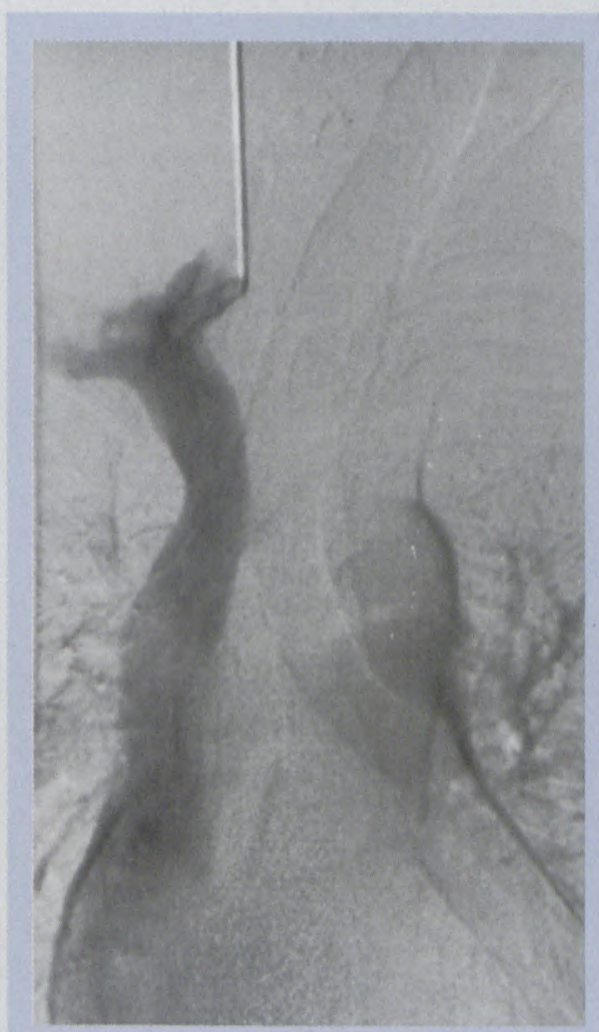

Fig. 5. Venogram to determine patency of internal jugular and superior vena cava.

should be present and a post placement venogram must always be done 


\section{SHORT REPORT}

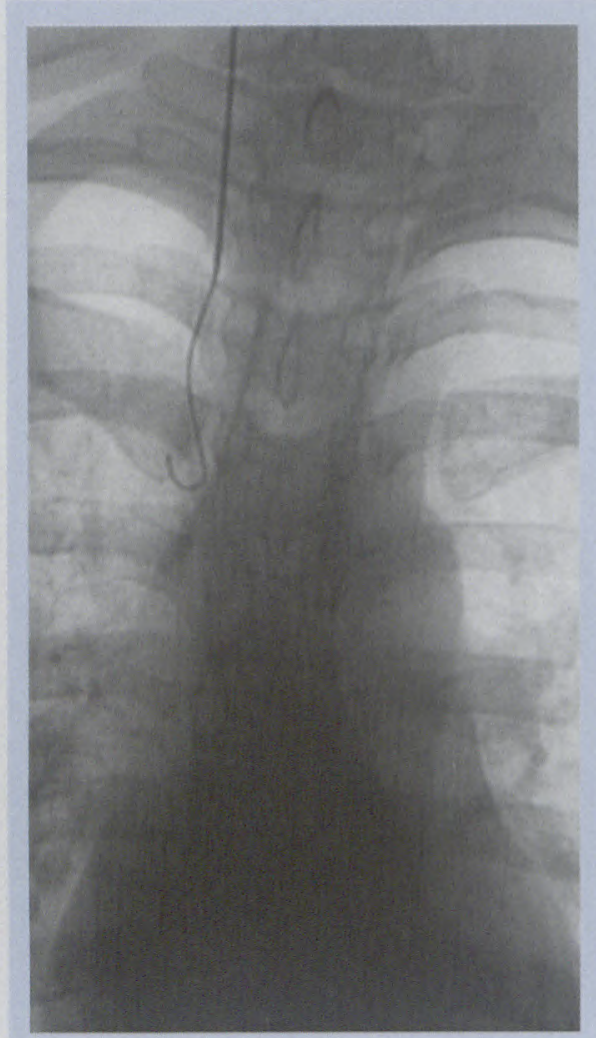

Fig. 6. Guidewire through dilator and peel-away catheter.

to prove the patency as well as the exact position of the line (Fig. 9).

Eight children and 52 adults underwent the procedure. The puncture areas included the right jugular $(N=35)$, left jugular $(N=14)$, right subclavian $(N=2)$, left subclavian $(N=1)$, left femoral $(N=4)$ and right femoral $(N=4)$. Two patients received multiple punctures, 2 lines were used for 1 patient, and 6 venograms were done. No sepsis was reported after the follow-ups of 1 week and 1 month.

As can be seen, the right internal jugular vein is the preferred site of puncture because of the easier placement of the guide wires and catheters from this position.

Placement of silicone catheters in an interventional unit is a good alternative to provide easily accessible ports for haemodialysis. There is a low complication rate, e.g. accidental

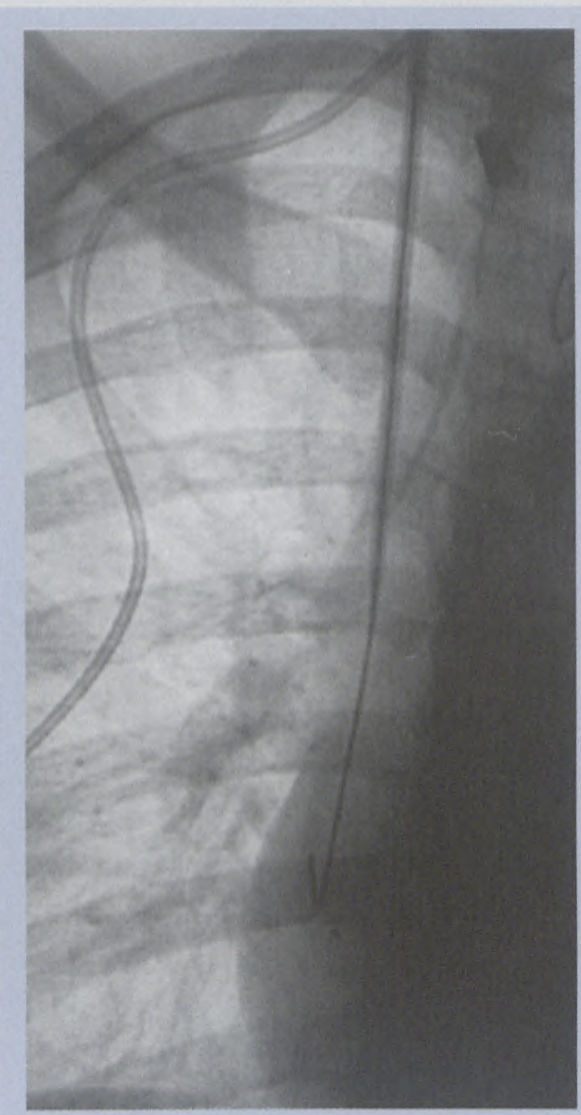

Fig. 7. Tunnelled catheter with dilator and peelaway sheath in place.

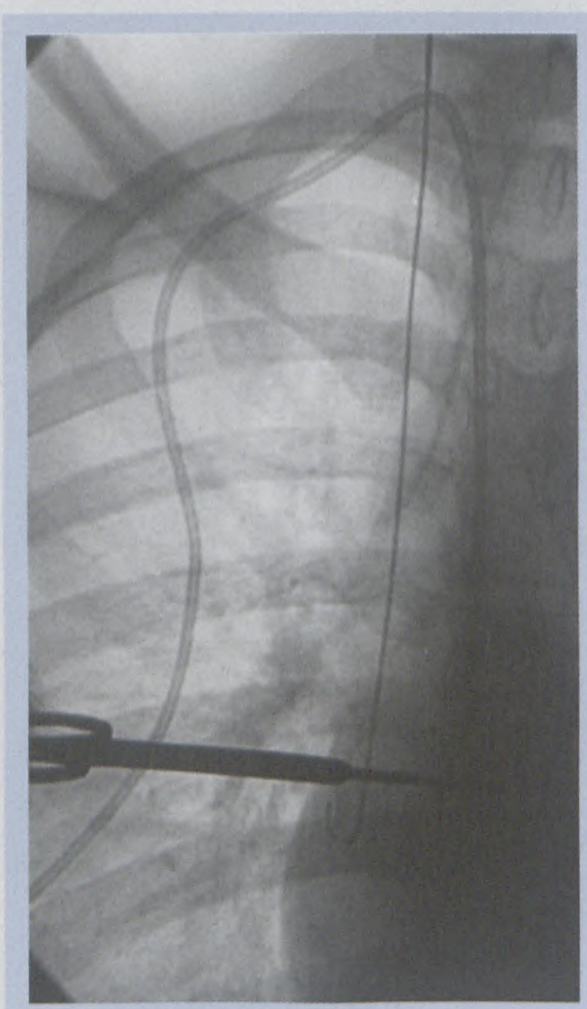

Fig. 8. Determine the length of the catheter.

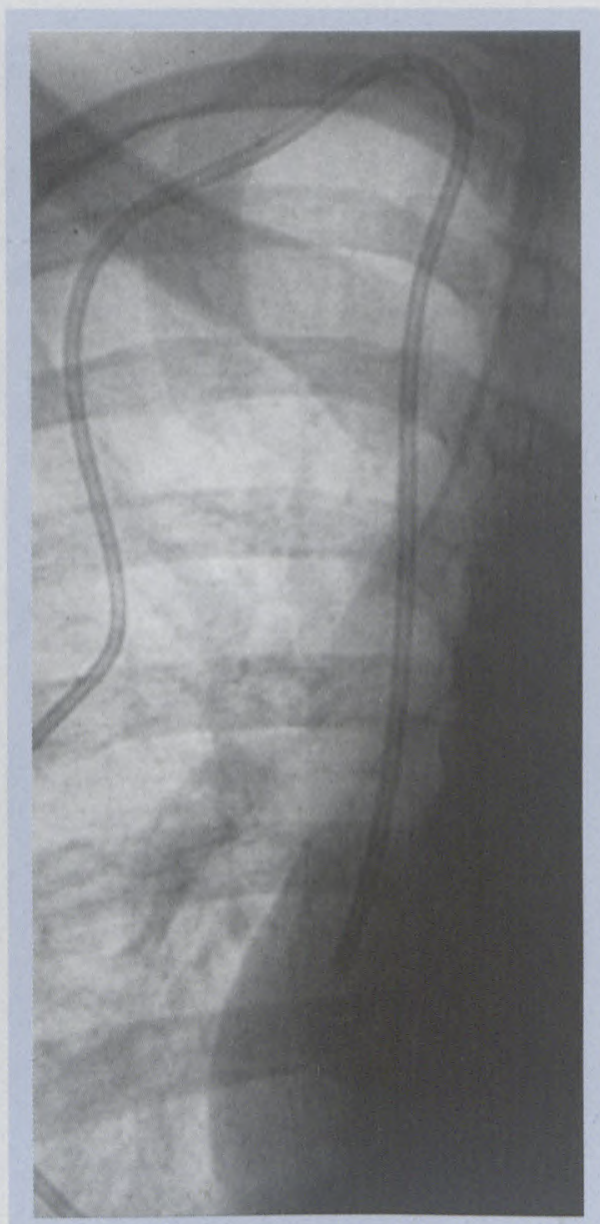

Fig. 9. Haemodialysis catheter in correct position.

puncture of the carotid artery or pneumothorax, because the whole procedure is done under direct fluoroscopy and ultrasound guidance. No sepsis within 1 month of the procedure was reported. 